INPLASY

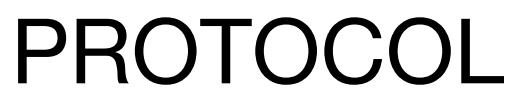

To cite: Teng et al.

Osteosarcopenia increasing the Risk of Falls, Fractures and Mortality? Inplasy protocol 202050111. doi:

10.37766/inplasy2020.5.0111

Received: 30 May 2020

Published: 30 May 2020

Corresponding author:

Sheng Lu

gwklsprof@163.com

Author Affiliation:

The First People's Hospital of Yunnan Province, Ku

Support: NFSC

Review Stage at time of this submission: Data analysis.

Conflicts of interest:

None.

\section{Osteosarcopenia increasing the Risk of Falls, Fractures and Mortality?}

Teng, Z1; Zhu, Y2; Yang, L3; Teng, Y4; Hao, Q5; Long, Q6; Zeng, Y7; Lu, S8.

Review question / Objective: Dose osteosarcopenia increasing the Risk of Falls, Fractures and Mortality? Condition being studied: Osteosarcopenia.

Information sources: PubMed, Embase and Cochrane Central Register of Controlled Trials (from their inception to May 25, 2020).

INPLASY registration number: This protocol was registered with the International Platform of Registered Systematic Review and Meta-Analysis Protocols (INPLASY) on 30 May 2020 and was last updated on 30 May 2020 (registration number INPLASY202050111).

\section{INTRODUCTION}

Review question / Objective: Dose osteosarcopenia increasing the Risk of Falls, Fractures and Mortality?

Condition being studied: Osteosarcopenia.

\section{METHODS}

Participant or population: Participant with or without osteoscarcopenia.
Intervention: Osteosarcopenia.

Comparator: Participant without
osteoscarcopenia.

Study designs to be included: Cohort study Cross-sectional study.

Eligibility criteria: Studies that either directly reported risk estimates (relative risks [RRs], odds ratios [ORs], or hazard ratios [HRs]) with $95 \%$ confidence intervals 
(CIs) or provided sufficient data to calculate these were included.

Information sources: PubMed, Embase and Cochrane Central Register of Controlled Trials (from their inception to May 25, 2020).

Main outcome(s): A meta-analysis of 13 studies indicated that osteosarcopenia significantly increased the fracture risk (OR, 2.72 [95\% CI, 2.11-3.52]).

Quality assessment / Risk of bias analysis: The quality of studies were assessed based on the Strengthening the Reporting of Observational Studies in Epidemiology (STROBE) statement.

Strategy of data synthesis: The quality of studies were assessed based on the Strengthening the Reporting of Observational Studies in Epidemiology (STROBE) statement.

Subgroup analysis: We performed subgroup analyses by sex, region, type of study design, sample size (we divided the included studies into three groups by the number of the participants(n) : $n \geq 1000$, $500 \leq n<1000$, and $n<500$ ), and osteosarcopenia type (sarcopenia plus osteoporosis, sarcopenia plus osteopenia, and sarcopenia plus osteoporosis/ osteopenia).

Sensibility analysis: Sensitivity analysis was conducted (by excluding each single study in turn) to estimate the influence of each individual study on the pooled results, and were conducted by excluding studies from Australia, cross-sectional studies, and studies of smaller sample size $(<500$ participants) to estimate the influence on the pooled results.

Country(ies) involved: China Australia Chile United State Switzerland Netherlands Korea Japan.

Keywords: Osteosarcopenia; Falls; Fractures; Mortality.
Contributions of each author:

Author 1 - Zhaowei Teng.

Author 2 - Yun Zhu.

Author 3 - Lirong Yang.

Author 4 - Yirong Teng.

Author 5 - Qinggang Hao.

Author 6 - Qing Long.

Author 7 - Yong Zeng.

Author 8 - Sheng Lu. 